\title{
Analytical model to estimate the performance of shuttle-based storage and retrieval systems with class-based storage policy
}

\author{
Michael Eder ${ }^{1}$
}

Received: 12 August 2019 / Accepted: 24 January 2020 / Published online: 14 March 2020

(C) The Author(s) 2020

\begin{abstract}
This paper presents a method to determine the performance of shuttle-based storage and retrieval systems (SBS/RS) with tiercaptive single-aisle shuttles and class-based storage policy. The use of this approach takes place both in the design process of SBS/RS and in the redesign process of SBS/RS. With this approach, it is possible to evaluate performance improvement by applying a class-based storage policy. Another beneficial scope of application of this approach is the evaluation of the placement of different classes throughout the rack to achieve the maximum throughput. The basis of this calculation method was a continuous-time open-queueing model with limited capacity. The cycle times of lifts and shuttles, as determined by a spatial value approach combined with a probability-based approach to mention the storage policy, could be directly used in the presented method with their time distributions. The data used herein were provided by a European material handling provider. An example was presented to outline how this calculation model can be used for optimizing the already existing SBS/RS via the application of a class-based storage policy. Through the example, depending on the configuration of the policy, applying the class-based storage to an existing SBS/RS would increase the throughput by up to twice the throughput without class-based storage policy.
\end{abstract}

Keywords Automated warehouse - Shuttle-based storage and retrieval system - Analytical and numerical modelling · Performance analysis · Class-based storage policy

\section{Introduction}

Global supply chain development towards a greater product variety and short response times have created new automated storage and retrieval systems (AS/RS) called shuttle-based storage/retrieval systems (SBS/RS) [1, 1114]. Typically, a tier-captive SBS/RS comprises multiple tiers with shuttles dedicated for each level. The shuttles cannot leave their tiers and fulfil the horizontal movement of the tote. The vertical movement is accomplished by lifts in front of the rack. Between these two subsystems for the lifts and shuttles, there are buffer slots in each tier, which describes the certain independence that exists between both systems. Moreover, these systems reach high levels of performance that can be quantified by their

Michael Eder

michael.e307.eder@tuwien.ac.at

1 Institute for Engineering Design and Product Development, Vienna University of Technology, Getreidemarkt 9, 1060, Wien, Austria achieved throughput. The maximum throughput is normally determined by the geometrical and technical specifications of the system. For instance, one way to achieve a higher throughput with a SBS/RS is to change the storage management policy into a class-based storage, which is the context of this paper. The advantage of switching from a uniform storage policy to a class-based storage policy is that it enables higher throughputs with the same physical parameters of the SBS/RS. There are two main reasons for doing this, the first being that the requirements for the SBS/RS change over time and the required performance is no longer achievable. A cost-effective way to increase throughput is to use a class-based storage policy. The second reason for using a class-based storage policy is that, when designing a new SBS/RS, the required performance is so high that a uniform storage policy cannot achieve the required throughput, and a class-based storage policy is an efficient way to achieve the required performance. For reasons of closeness to reality, the presented approach is presumed to be discrete in space and continuous in time.

The purpose of this document is to provide a decision tool that can quickly and accurately assess the throughput 
of a SBS/RS with class-based storage policy. This approach should be used both in the design process of a new SBS/RS and in the redesign process of an existing SBS/RS to achieve higher performance.

The main contribution of this paper is to present an analytical approach to discussing SBS/RS with class-based storage policies. This approach can be used directly in the design process of SBS/RS. In addition, this article describes how to apply class-based storage policies to SBS/RS and the resulting changes in throughput.

This paper is organized as follows. The concept is explained in the literature provided in Section 2. The SBS/RS in question and the underlying assumptions are described in Section 3. An analytical approach for treating the system, especially the different possibilities of classbased storage and how to treat these, is discussed in Section 4. In Section 5, a numerical study is carried out to demonstrate the influence of the class-based storage on the throughput. The parameters used in this study are sourced from a European material handling provider. Finally, the findings of this paper are summed up in Section 6, where an outlook on the future of this research is included.

\section{Literature review}

Existing research on tier-captive shuttle-systems with random storage assignment is substantial. Eder [2] asserted two possible ways of discussing SBS/RS: one using discrete event simulation (DES) and the other via an analytical approach to examine such systems. Nonetheless, there have been rare accounts discussing class-based storage policies in SBS/RS. Kuo et al. [10], for instance, discussed the use of a queueing network to investigate a SBS/RS with class-based storage. Such approach though was limited; although it was only able to calculate the waiting times within one handling cycle, the maximum throughput could not be extracted [2]. Ekren et al. [6] presented an approach that treated, or rather, simulated class-based storage policies in SBS/RS. Kriehn et al. [9] conducted another simulation study on the same topic in an attempt to optimize the class-based storage policy and to quantify the reachable benefit of the storage policy.

Likewise, Schloz et al.[15] presented a different way of discussing storage policies in SBS/RS by developing an optimization approach to find the best places for the different classes in the rack.

On the contrary, there were more studies on SBS/RS with random storage. Literature [2-5] describes the manner in which the system is closest to its real behavior. More importantly, Eder [2] additionally used a spatially discrete approximation, which likewise corresponds to reality. An overview of all existing literature on SBS/RS is presented in Table 1. As can be seen in this table, there are some articles that deal with class-based storage policies, but there is no analytical approach that determines the achievable throughput. On the other hand, there are papers that provide analytical decision tools, but these approaches apply only to uniform storage policy. This is the reason for the research gap in analytical decision tools for determining the maximum throughput of SBS / RS with a class-based storage policy. This study specifically adopted and advanced the main idea in Eder [2] through consideration of several probability based approaches within the class-based storage policy.

\section{System description}

The system under investigation is a tier-captive single-aisle SBS/RS as displayed in Fig. 1. A lift can be seen in front

Table 1 An overview of the literature on SBS/RS

\begin{tabular}{|c|c|c|c|c|c|}
\hline Paper & Type of system & Storage policy & Model $^{1}$ & Storage depth & $\begin{array}{l}\text { Ability to determine } \\
\text { the max. throughput }\end{array}$ \\
\hline Kuo et al. [10] & Tier-captive & Class-based & QN & Single deep & No \\
\hline Marchet et al. [13] & Tier-captive & Uniform & QN & Single deep & No \\
\hline Ekren et al. [6] & Tier-captive & Class-based & SIM & Single deep & Yes \\
\hline Eder et al. [3] & Tier-captive & Uniform & SQLC & Double deep & Yes \\
\hline Eder et al.[4] & Tier-captive & Uniform & SQLC & Single deep & Yes \\
\hline Schloz et al. [15] & Tier-captive & Class-based & OPT & Single deep & No \\
\hline Eder et al. [5] & Tier-captive & Uniform & SQLC & Single deep & Yes \\
\hline Kriehn et al. [9] & Tier-captive & Class-based & SIM & Single deep & Yes \\
\hline Eder [2] & Tier-captive & Uniform & SQLC & Single deep & Yes \\
\hline
\end{tabular}

${ }^{1} C T M$, cycle time model; $Q N$, queueing network; $S I M$, simulation; $S Q$, single queue; $S Q L C$, single queue with limited capacity; $O P T$, optimization approach 
Fig. 1 Shuttle-system [2]

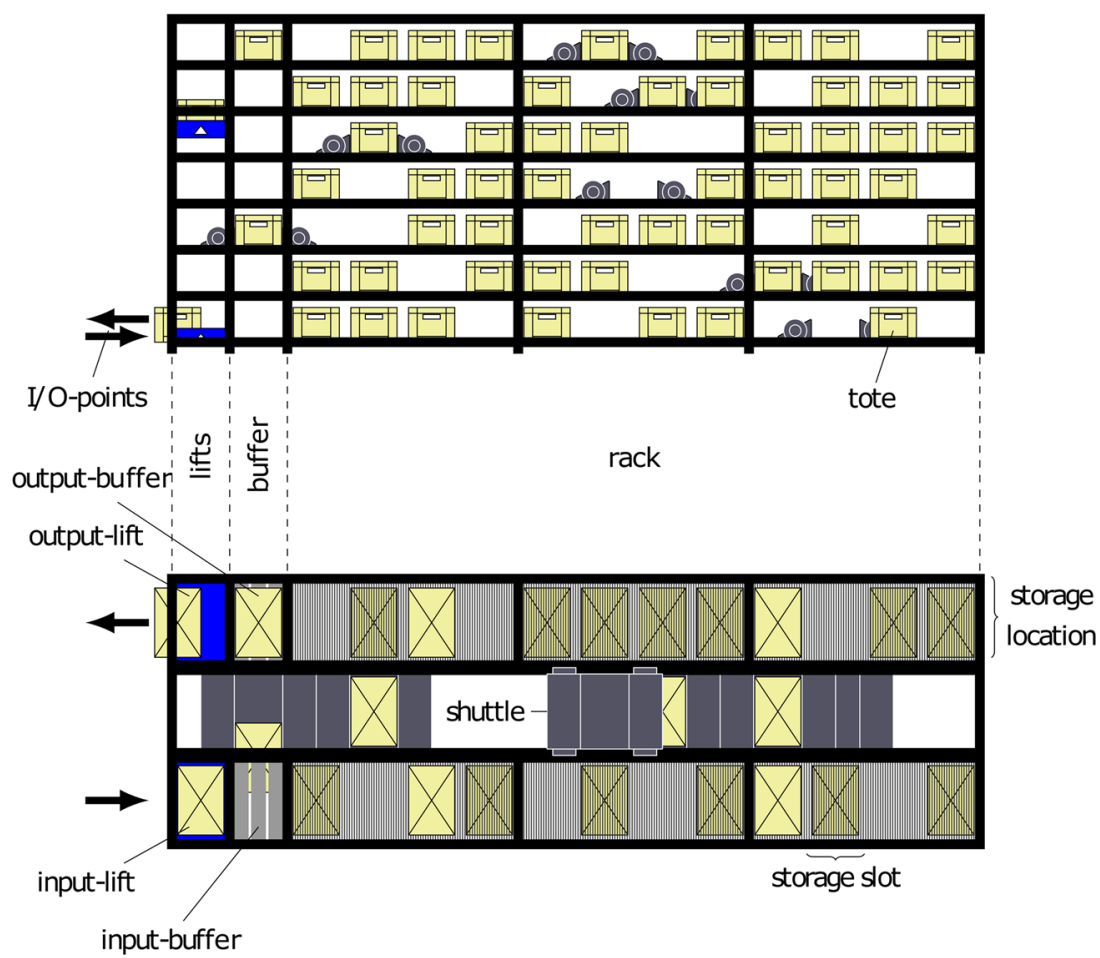

of each rack for totes transport from the input/output (I/O) point to the tier and back. Basically, these I/O points are located on the first tier in front of the lifts. There are also buffers between the lifts and shuttles. Each shuttle is assigned to one tier in one aisle, which means that there are as many vehicles as tiers in a rack. Furthermore, these vehicles can transport one tote at a time along the aisle. The racks are single-deep and double sided. Moreover, each storage location can hold one tote [2].

Assumptions (and some notations) proposed in this paper were similar to those for a SBS/RS produced by a European material handling provider. The first eight assumptions have also been published under Eder and Eder et al. [2-5]:

1. Both lifts realize transactions in a single-command cycle under a first-come-first-served (FCFS) rule; thus, one lift is for input and the other is for output.

2. The shuttles realize the transactions in single and double cycles under a FCFS rule.

3. The dwelling point for the input lift is the $\mathrm{I} / \mathrm{O}$ point.

4. The dwelling point for the output lift is in front of the tier where the next tote has to be restored.

5. The dwelling point of the shuttle is in front of the buffer-slots.

6. The lifts and shuttles accelerate/decelerate with constant acceleration/deceleration. In case the acceleration/deceleration is not constant, the actual acceleration/deceleration must be transferred to an acceleration/deceleration which gives the same behaviour as the actual acceleration/deceleration of the SBS/RS.
7. There is no difference in time between the transfer of totes to or from the lifts. If there is a difference in the real system, this has no effect on the calculation since only the sum of times is used for loading and unloading.

8. There are always totes on the I/O point waiting to be stored. This assumption is necessary to achieve the maximum throughput. Otherwise, the input lift must wait for incoming totes, which would affect the possible performance of the SBS / RS under consideration.

9. The order of the totes to be re-stored next is evenly distributed among all stored totes of the same class.

10. The order of the totes from different classes is as given by the storage policy.

11. The assignment of the class to a tote is static and can not be changed while the tote is stored.

Assumptions 9 through 11 are the definitions of class-based storage. The parameters of these assumptions result from the classification of the data of a warehouse management system.

\section{Analytical approach}

A single-aisle model was deemed fit for determining the performance of SBS/RS, as the storage and retrieval transactions among all aisles were evenly distributed [7, 8, 13].

For this study, the presented approach is based on the approach by Eder [2], which employs an open queueing model with limited capacity $(\mathrm{M}|\mathrm{G}| 1 \mid \mathrm{K})$ and with three main parts determining the throughput, namely the interarrival time to 
Table 2 Notation of the tier-captive single-aisle AVS/RS

\begin{tabular}{|c|c|}
\hline$\Delta x$ & Distance between two storage slots \\
\hline$\Delta y$ & Distance between two tiers \\
\hline$\vartheta$ & Throughput of a single aisle \\
\hline$\vartheta_{\text {tier }}$ & Throughput of a single tier \\
\hline$\vartheta_{\text {tier }_{N}} / \vartheta_{\text {tier }_{X}}$ & Throughput of a single tier in zone $\mathrm{N} /$ area $\mathrm{X}$ \\
\hline$\rho$ & Utilization rate of the shuttle \\
\hline$\rho_{N} / \rho_{X}$ & Utilization rate of the shuttle in zone $\mathrm{N}$ / area $\mathrm{X}$ \\
\hline A & Number of aisles \\
\hline$a_{\text {lift }}$ & Lift acceleration/deceleration rate \\
\hline$a_{\text {shuttle }}$ & Shuttle acceleration/deceleration rate \\
\hline$K$ & Capacity of the queueing system \\
\hline$l_{I / O}$ & Vertical distance between the first tier and the $\mathrm{I} / \mathrm{O}$ point \\
\hline$n_{\text {buf }}$ & Number of buffers on each side of the aisle per tier \\
\hline$n_{\text {slot }}$ & Number of slots on each side of the aisle per tier \\
\hline$n_{\text {slot }_{N}}$ & Number of slots on each side for each zone $\mathrm{N}$ within a tier \\
\hline$n_{\text {slot }}{ }_{N X}$ & Number of slots on each side for each zone $\mathrm{N}$ within a tier from area $\mathrm{X}$ \\
\hline$n_{\text {tier }}$ & Number of tiers \\
\hline$n_{\text {tier }} / n_{\text {tier }}$ & Number of tiers in zone $\mathrm{N} /$ area $\mathrm{X}$ \\
\hline$p_{0}$ & Probability of emptiness of a queueing system \\
\hline$p_{0_{N}} / p_{0_{X}}$ & Probability of emptiness of a queueing system in zone N/ area X \\
\hline$p_{k}$ & Blocking probability of a queueing system \\
\hline$p_{k_{N}} / p_{k_{X}}$ & Blocking probability of a queueing system in zone $\mathrm{N}$ / area $\mathrm{X}$ \\
\hline$t_{A}$ & Interarrival time to a tier \\
\hline$t_{A_{N}} / t_{A_{X}}$ & Interarrival time to a tier of zone $\mathrm{N} /$ area $\mathrm{X}$ \\
\hline$t_{t_{L}}$ & Time to transfer a tote to and from the lift \\
\hline$t_{t_{S}}$ & Time to transfer a tote to and from the shuttle \\
\hline$t_{\text {lift }}$ & Cycle time of a lift for a single-command cycle \\
\hline$t_{R_{L}}$ & Time to travel of a lift at single-command cycle \\
\hline$t_{R_{S} S C}$ & Time to travel of a shuttle at single-command cycle \\
\hline$t_{R_{-} D C}$ & Time to travel of a shuttle at dual-command cycle \\
\hline$t_{S}$ & Service time of a shuttle \\
\hline$t_{S_{S C}}$ & Service time of a shuttle at single-command cycle \\
\hline$t_{S_{D C}}$ & Service time of a shuttle at dual-command cycle \\
\hline$t_{\text {shuttlesC }}$ & Cycle time of a shuttle for a single-command cycle \\
\hline$t_{\text {shuttle }_{D C}}$ & Cycle time of a shuttle for a dual-command cycle \\
\hline$s$ & Coefficient of variation of the cycle times \\
\hline $\mathrm{x} s_{N} / s_{X}$ & Coefficient of variation of the cycle times in zone $\mathrm{N}$ / area $\mathrm{X}$ \\
\hline$v_{l i f t}$ & Lift velocity \\
\hline$v_{\text {shuttle }}$ & Shuttle velocity \\
\hline$w_{N} / w_{X}$ & Probability of order a tote in zone N / area X \\
\hline$w_{N X}$ & Probability that a tote from zone $\mathrm{N}$ is situated in area $\mathrm{X}$ \\
\hline
\end{tabular}

one single tier, the service time of the shuttles, and the open queueing model $\mathrm{M}|\mathrm{G}| 1 \mid \mathrm{K}$. The open queuing model with limited capacity $(\mathrm{M}|\mathrm{G}| 1 \mid \mathrm{K})$ is used because this approach takes into account the interactions between shuttles and lifts. These interactions lead to waiting times of lifts and shuttles which directly affects the achievable throughput. To adapt this approach, modifications have been made to all three parts.
In this chapter, the approach was initially enacted via the procedure in Eder [2] for an easier comprehension of the adaptation. Next, zoning in the horizontal and vertical directions was applied, followed by the respective discussion. The notations used in this analytical approach are listed in Table 2. The approach procedure was as follows (Fig. 2): 


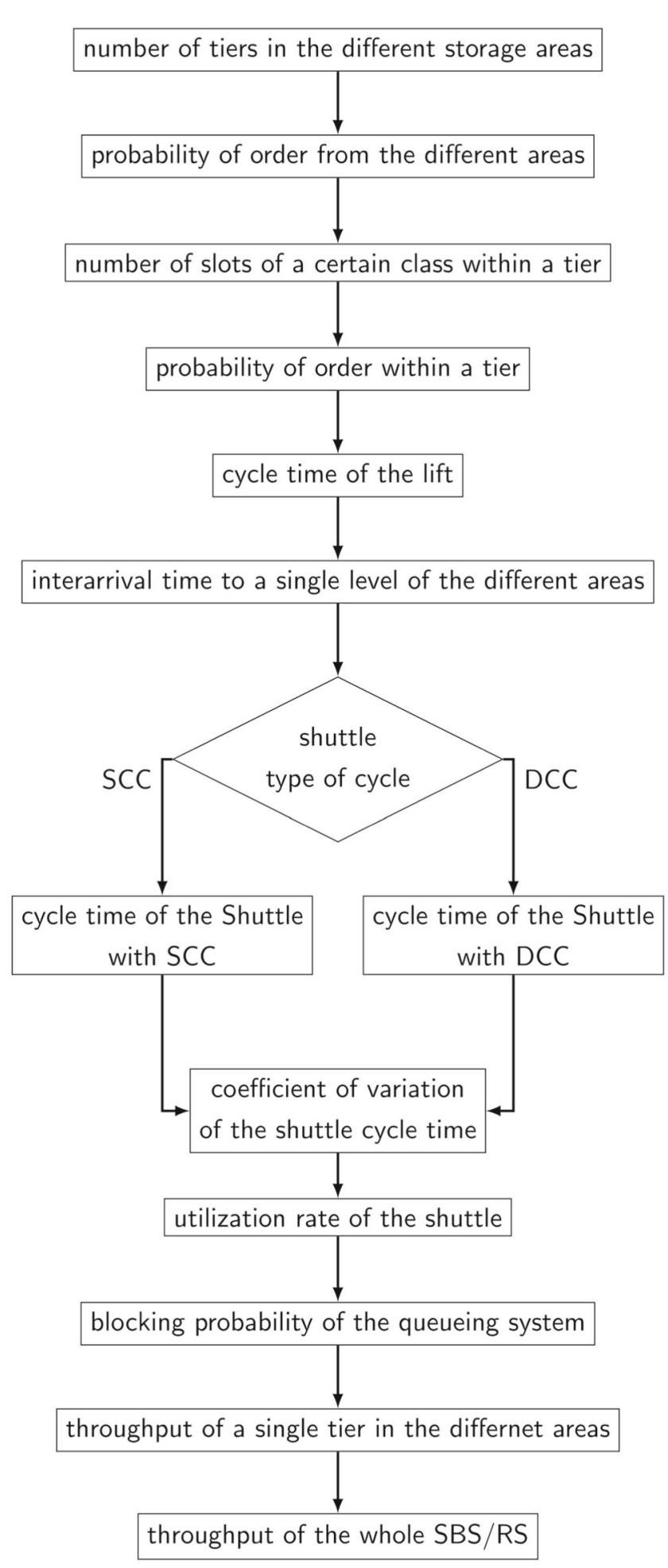

Fig. 2 Flowchart of the procedure used for the approach proposed in the study

- The number of tiers in the different storage areas (only zoning in vertical direction and with combined zoning in vertical and horizontal directions) was determined.
- Order probability from the different areas (only zoning in vertical direction and with combined zoning in vertical and horizontal directions) was determined.

- The number of slots of the different classes within a tier (only zoning in horizontal direction and with combined zoning in vertical and horizontal directions) was determined.

- Order probability within a tier of the different areas (only zoning in horizontal direction and with combined zoning in vertical and horizontal directions) was determined.

- The interarrival time of the totes in each storage level was determined depending on the zoning.

- Service time within one storage level of the shuttles was measured.

- The throughput when using the open queueing model $\mathrm{M}|\mathrm{G}| 1 \mid \mathrm{K}$ was calculated.

The class-based storage parameters such as the number of containers from the different zones $\left(n_{N}\right)$ or the probability of ordering from a zone $\left(w_{N}\right)$ must be determined by classifying the data of a warehouse management system.

\subsection{Throughput calculation without zoning}

The throughput of a shuttle system without zoning was calculated herein based on Eder [2].

\subsubsection{Interarrival time}

Firstly, the interarrival time was calculated as determined by the lifts (Eq. 1); here, the time for the ride and the times for transferring the tote to and from the lift were required [2],

$t_{\text {lift }}=2 \cdot t_{R_{L}}+t_{t_{L}}$

The mean time for the ride is as follows:

$t_{R_{L}}=\frac{1}{n_{\text {tier }}} \sum_{k=1}^{n_{\text {tier }}} \mathbf{t}(|(k-1) \cdot \Delta y|)$

Considering the fact that the lifts will not reach their maximum speed within small distances, the function $\mathbf{t}(l)$ has to be split into two ranges, with the first part for distances being less than $l<\frac{v^{2}}{a}$,

$\mathbf{t}(l)=2 \sqrt{\frac{l}{a_{l i f t}}}$

while the other part was for larger distances,

$\mathbf{t}(l)=\frac{l}{v_{\text {lift }}}+\frac{v_{\text {lift }}}{a_{\text {lift }}}$

Accordingly, Eq. 1 represents the cycle time of the lift for one single-command lift cycle. Here, the interarrival time is this cycle time times the number of tiers, as follows:

$t_{A}=t_{\text {lift }} \cdot n_{\text {tier }}$ 
Moreover, this equation incorporates the fact that one lift serves all the tiers of an aisle.

\subsubsection{Service time}

The second requirement for determining the throughput was the shuttle service time. It is obtained in the same process as the cycle times of the lifts. Transport times from A to $\mathrm{B}$ and tranferring times of the totes to and from the shuttle were measured as well [2].

Single-command cycle: The single-command cycle is best represended by [2]

$$
t_{\text {shuttlesC }}=2 \cdot t_{R_{S} S C}+t_{t_{S}}
$$

and the mean time for the ride is due to the following:

$$
t_{R_{S} S C}=\frac{1}{n_{\text {slot }}} \sum_{k=1}^{n_{\text {slot }}} \mathbf{t}(k \cdot \Delta x)
$$

Equations 3 and 4 should be used for determining the times depending on the distances.

The service time of the shuttles is given by the following:

$t_{S_{S C}}=2 \cdot t_{\text {shuttle }}$ SC

the multiplier 2 reflects that the reference cycle in this paper is a dual handling cycle.

Dual-command cycle: Accordingly, the dual-command cycle is represented by the following:

$$
t_{\text {shuttle }_{D C}}=2 \cdot t_{R_{S_{S} S C}}+t_{R_{S_{-} D C}}+2 \cdot t_{t_{S}}
$$

The first term in Eq. 9 is the same as for the single cycle, while the second term stands for the ride between the slot where a tote is transferred from the shuttle and the slot, where the next tote, that shall be retrieved, is located.

The mean time for this is due to

$t_{R_{S-D C}}=\frac{1}{n_{\text {slot }}^{2}} \sum_{k=1}^{n_{\text {slot }}} \sum_{l=1}^{n_{\text {slot }}} \mathbf{t}(|(k \cdot \Delta x-l \cdot \Delta x)|)$

Moreover, the service time of the shuttles with double cycle is given by the following:

$t_{S_{D C}}=t_{\text {shuttle }_{D C}}$

\subsubsection{Open queueing model M | G | 1 | K}

A time-continuous open-queueing model with limited capacity was used for evaluating the influence of the buffers and the influence of the interaction between lifts and shuttles. Through this model, the throughput of one single tier $\vartheta_{\text {tier }}$ can be calculated with [2]

$\vartheta_{t i e r}=\frac{1}{t_{A}} \cdot\left(1-p_{k}\right)$ $\vartheta_{\text {tier }}=\frac{1}{t_{S}} \cdot\left(1-p_{0}\right)$

Two methods can be employed to determine the throughput. The first method is by interarrival time and blocking probability (Eq. 12), the second by using the service time and the probability of emptiness (Eq. 13). Blocking probability means that the system is completely filled so that no tote can enter the system. Applied to a shuttle system this means that the lift has to wait for an empty space in the input-buffer. The probability of emptiness implies that the server has to wait because there is no tote in the waiting area. In SBS/RS, it means that the shuttle has to wait for a tote.

The throughput of an aisle is equal to the throughput of one tier multiplied by the number of tiers

$\vartheta=\vartheta_{\text {tier }} \cdot n_{\text {tier }}$

and the blocking probability of the queueing system can be calculated through [16]

$p_{k}=\frac{\rho^{\frac{\sqrt{\rho} \cdot s^{2}-\sqrt{\rho}+2 K}{2+\sqrt{\rho} \cdot s^{2}-\sqrt{\rho}}} \cdot(\rho-1)}{\rho^{2 \cdot \frac{\sqrt{\rho} \cdot s^{2}-\sqrt{\rho}+K+1}{2+\sqrt{\rho} \cdot s^{2}-\sqrt{\rho}}}-1}$

Equation 15 is a seemingly complex equation, but in reality, it only contains three different arguments. The main argument is the utilization rate of the service station (=shuttle), which contains the interarrival time defining the lift and the service time of the shuttle which can be obtained by the expression

$\rho=\frac{t_{S}}{t_{A}}$

The second argument is $K$, which represents the capacity of the queueing system. It is essentially the summation of the number of buffers and the capacity of the shuttles in the treated SBS/RS, which is always one tote.

$K=n_{b u f}+1$

The third and last argument is $s_{N}$, which is the coefficient of variation of the service process. It is easily calculated upon simplification of Eqs. 6 and 9, with the same equations for different distances as Eqs. 3 and 4. Subsequently, the coefficient of variation for a shuttle that simplifies the calculation single handling cycle is [5]

$s=\frac{\sqrt{\frac{\left(2 \cdot \frac{n_{\text {slot }} \cdot \Delta x}{v_{\text {shuttle }}}\right)^{2}}{12}}}{t_{S}}$

and for the double handling cycle with [5]

$s=\frac{\sqrt{\frac{\left(2 \cdot \frac{n_{\text {slot }} \cdot \Delta x}{v_{\text {shuttle }}}\right)^{2}}{18}}}{t_{S}}$ 
Respectively, the probability of emptiness contains the same arguments (as in the blocking probability) as expressed by [16]:

$$
p_{0}=\frac{\rho-1}{\rho^{2 \cdot \frac{\sqrt{\rho} \cdot s^{2}-\sqrt{\rho}+K+1}{2+\sqrt{\rho} \cdot s^{2}-\sqrt{\rho}}}-1}
$$

\subsection{Zoning in horizontal direction}

Figure 3 illustrates the SBS/RS treatment with zoning in horizontal direction, which is the first zoning section. There are different zones throughout the rack length. For the throughput calculation this kind of zoning only influences the service time of the shuttles. Moreover, interarrival time of the lift is the same, as all tiers have the same probability of arrival. In addition, the open queueing system can be calculated the same way as without zones.

Horizontal zoning is defined as the number of slots for each zone $N$ within a tier as in,

$n_{\text {slot }}=w_{N} \cdot n_{\text {slot }}$

\subsubsection{Interarrival time}

The interarrival time to the tiers is basically the same as the interarrival time without zoning (see Section 4.1.1), mainly because there are no different zones in the vertical direction.

\subsubsection{Service time}

In the horizontal direction, the rack is split into different zones depending on the class of the tote, causing the service time of the shuttles to be different from that in a rack without zoning.

Single-command cycle: The main equation for zoning in horizontal direction is similar to Eq. 6 for the service time without zoning, as given by

$$
t_{S}=t_{\text {shuttle }_{S C}}=2 \cdot t_{R_{S} S C}+t_{t_{S}}
$$

The difference from zoning to no zoning is the time for ride $t_{R_{S_{-} S C}}$. For two zones in horizontal direction $t_{R_{S_{-} S C}}$ is given by

$$
\begin{aligned}
t_{R_{S-S C}}= & w_{1} \cdot \frac{1}{n_{\text {slot }_{1}}} \sum_{k=1}^{n_{\text {slot }}} \mathbf{t}(k \cdot \Delta y) \\
& +w_{2} \cdot \frac{1}{n_{\text {slot }_{2}}} \sum_{k=1}^{n_{\text {slot }}} \mathbf{t}\left(\left|n_{\text {slot }_{1}} \cdot \Delta x+k \cdot \Delta x\right|\right)
\end{aligned}
$$

The first term in Eq. 23 describes the time for the ride to zone 1 . Here, the first part $w_{1}$ refers to the probability of ordering a tote from class 1 , while the second part describes the mean time for the ride to zone 1 . The second term stands for the ride to zone 2 with the probability of the order from class 2 . The difference between the two terms is in the mean time for the ride, with the second term including the ride across zone $1\left(n_{\text {slot }_{1}} \cdot \Delta x\right)$. For higher numbers of zones, the equation expands to

$$
\begin{aligned}
& t_{R_{S} S C}=\sum_{N=1}^{\text {Zones }} w_{N} \cdot \frac{1}{n_{\text {slot }_{N}}} \\
& \cdot \sum_{k=1}^{n_{\text {slot }}} \mathbf{t}\left(\left|\sum_{M=0}^{N-1} n_{\text {slot }_{M}} \cdot \Delta x+k \cdot \Delta x\right|\right)
\end{aligned}
$$

Fig. 3 Zoning in horizontal direction

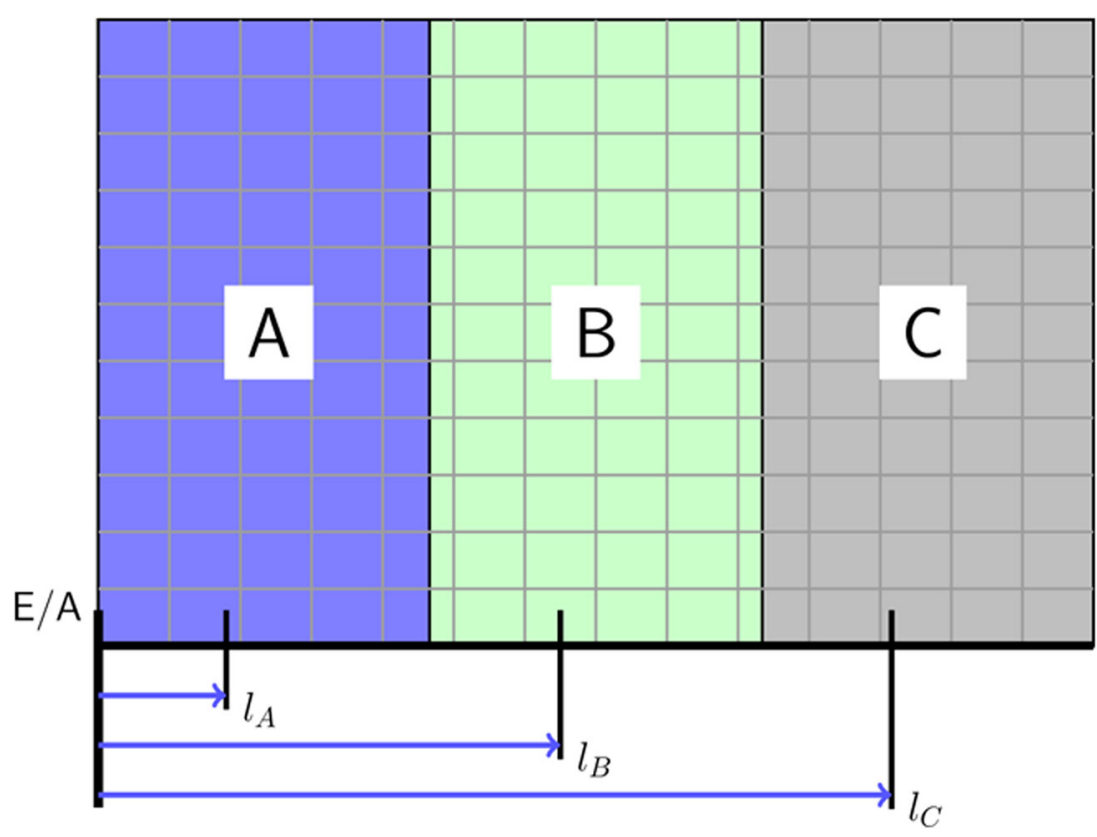


Dual-command cycle: The dual-command cycle can be expressed by

$$
t_{S}=t_{\text {shuttle }_{D C}}=2 \cdot t_{R_{S_{-} S C}}+t_{R_{S_{-} D C}}+2 \cdot t_{t_{S}}
$$

which is quite similar to Eq. 22 for the single-command cycle. The difference is the time for the ride between the storage location at the storage process and the location at the retrieval process, $t_{R_{S} D C}$. For two zones in horizontal direction the mean time for the ride between these two locations is given by

$$
\begin{aligned}
t_{R_{S} D C}= & \frac{w_{1}}{n_{\text {slot }_{1}}} \cdot \frac{w_{1}}{n_{\text {slot }_{1}}} \sum_{l=1}^{n_{\text {slot }_{1}}} \sum_{m=1}^{n_{\text {slot }}} \mathbf{t}(|l \cdot \Delta x-m \cdot \Delta x|) \\
+ & \cdot \frac{w_{1}}{n_{\text {slot }_{1}}} \cdot \frac{w_{2}}{n_{\text {slot }_{2}}} \\
& \cdot \sum_{l=1}^{n_{\text {slot }_{1}}} \sum_{m=1}^{n_{\text {slot }}} \mathbf{t}\left(\left|l \cdot \Delta x-m \cdot \Delta x-n_{\text {slot }} \cdot \Delta x\right|\right) \\
+ & \frac{w_{2}}{n_{\text {slot }_{2}}} \cdot \frac{w_{2}}{n_{\text {slot }_{2}}} \sum_{l=1}^{n_{\text {slot }}} \sum_{m=1}^{n_{\text {slot }}} \mathbf{t}(|l \cdot \Delta x-m \cdot \Delta x|)
\end{aligned}
$$

The first term in Eq. 26 describes the situation wherein the storage location at the storage process and at retrieval process are both situated in zone 1 and could be taken to equal Eq. 10 of the ride between two storage locations in the dual-command cycle of the shuttle without zoning. Accordingly, the second term involves two cases, namely (i) the storage location is in zone 1 while the retrieval location in zone 2, and (ii) the storage location is in zone 2 while the retrieval location in zone 1 . The third term stands for both locations in zone 2. For higher numbers of zones, the equation evolves to

$$
\begin{aligned}
& \sum_{J=1}^{t_{R_{S} D C}}= \\
& \sum_{K=1}^{\text {Zones }} \sum_{\text {Zones }} \frac{w_{J}}{n_{\text {slot }_{J}}} \frac{w_{K}}{n_{\text {slot }_{K}}} \\
& \cdot \sum_{l=1}^{n_{\text {slot }}} \sum_{m=1}^{n_{\text {slot }_{K}}} \mathbf{t}\left(\mid\left(l+\sum_{O=0}^{J-1} n_{\text {slot }_{O}}-m-\sum_{P=0}^{K-1} n_{\text {slot }_{P}} \mid\right) \cdot \Delta x\right)
\end{aligned}
$$

\subsubsection{Open queueing model M | G | 1 | K}

Here, the queueing model is the same as without zoning (see Section 4.1.3), practically because there is no zoning in vertical direction and thus tiers work the same manner. The only thing that has to be changed is the determination of the coefficient of variation of the service times, which is essentially obtained through numerical simulation.

\subsection{Zoning in vertical direction}

The second zoning type is zoning in vertical direction, as demonstrated in Fig. 4. Such zoning influences the calculation of interarrival time by the lift, and also the open queuing system that needs calculation for every zone in the SBS/RS.

Vertical zoning is defined as the number of tiers for each zone $N$ within an aisle,

$n_{\text {tier }}=w_{N} \cdot n_{\text {tier }}$

\subsubsection{Interarrival time}

The interarrival time of the lift changes with zoning in vertical direction. On the one hand, the cycle time of the lift changes; on the other hand, the transformation of the cycle times to the interarrival time changes.

Single-command cycle: The main equation for zoning in vertical direction is similar to the equation for the cycle time with no zoning Eq. 1 and is given by the following:

$t_{\text {lift }}=2 \cdot t_{R_{L_{-} S C}}+t_{t_{L}}$

The difference between zoning and no zoning is the time for the ride $t_{R_{L S C}}$. For two zones in horizontal direction, the time for the ride can be calculated through the following:

$$
\begin{aligned}
t_{R_{L_{-} S C}}= & w_{1} \cdot \frac{1}{n_{\text {tier }_{1}}} \sum_{k=1}^{n_{\text {tier }}} \mathbf{t}(|(k-1) \cdot \Delta y|) \\
& +w_{2} \cdot \frac{1}{n_{\text {tier }}} \sum_{k=1}^{n_{\text {tier }}} \mathbf{t} \mathbf{t}\left(\left|n_{\text {tier }} \cdot \Delta y+(k-1) \cdot \Delta y\right|\right)
\end{aligned}
$$

The first term in Eq. 30 is the time for the ride to zone 1. The first part, $w_{1}$, indicates the probability of ordering a tote from class 1, while the second part describes the mean time for the ride to zone 1 . The second term stands for the ride to zone 2 with the probability of the order from class 2 . The difference between both terms is in the mean time for the ride, where the second term includes the ride across zone $1\left(n_{t i e r_{1}} \cdot \Delta y\right)$. For higher numbers of zones, the equation becomes

$$
\begin{aligned}
& \sum_{N=1}^{t_{R_{L_{-} S C}}}= \\
& \sum_{\text {Zones }}^{n_{\text {tier }_{N}}} w_{N} \cdot \frac{1}{n_{\text {tier }_{N}}} \\
& \cdot \sum_{k=1}^{N-1} \mathbf{t}\left(\left|l_{I / O}+(k-1) \cdot \Delta y\right|+\sum_{M=0}^{N-1} n_{\text {tier }_{M}} \cdot \Delta y\right)
\end{aligned}
$$

Transformation of the lifts' cycle time to the interarrival time: The cycle time of the lifts can be transformed into the interarrival time without zoning by multiplying the cycle time with the number of tiers in the system, as depicted in Eq. 5. With zoning in vertical direction, the conversion 
Fig. 4 Zoning in vertical direction

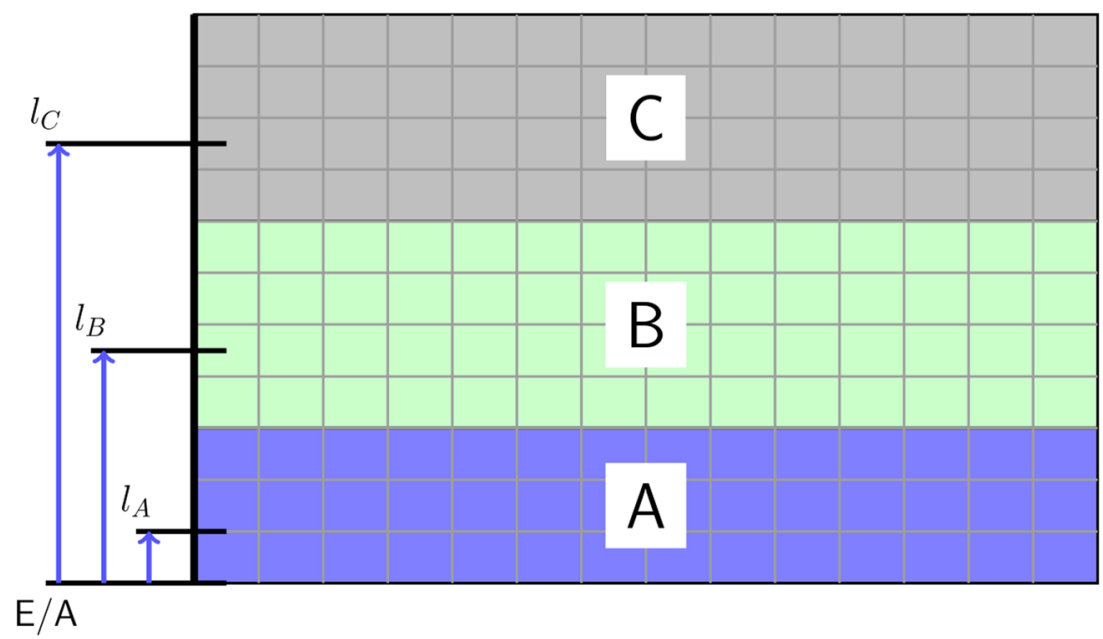

is a bit different. Here, the cycle time has to be multiplied by the number of tiers in the respective zone, and then it has to be divided by the probability of selection of this zone, so as to take the class-based storage policy into account,

$t_{A_{N}}=t_{\text {lift }} \cdot \frac{n_{\text {tier }}}{w_{N}}$

\subsubsection{Service time for a single depth rack}

The service time for zoning in vertical direction is the same for all the zones. Thus, the calculation of this time is as for SBS/RS without zoning (see Section 4.1.2).

\subsubsection{Open queueing model $\mathrm{M}$ | $\mathrm{G}$ | 1 | K}

The calculation of the open queueing system with limited capacity with zoning in vertical direction is different from the calculation without zoning. With zoning in vertical direction the tiers are split up into different zones. Therefore, the queueing system has to be calculated several times for each zone. The equations for determining the throughput of one single tier with $N$ as the number of the zone include

$\vartheta_{\text {tier }_{N}}=\frac{1}{t_{A_{N}}} \cdot\left(1-p_{k_{N}}\right)$

$\vartheta_{\text {tier }_{N}}=\frac{1}{t_{S_{N}}} \cdot\left(1-p_{0_{N}}\right)$

Here, the throughput of an aisle is the summation over the different zones of the throughput of one tier in the respective zone multiplied by the number of tiers in this zone, as

$\vartheta=\sum_{N=1}^{\text {Zones }} \vartheta_{\text {tier }_{N}} \cdot n_{\text {tier }_{N}}$
For each zone $N$, the blocking probability of a queueing system can be found with the expression,

$$
p_{k_{N}}=\frac{\rho_{N}^{\frac{\sqrt{\rho_{N}} \cdot s_{N}^{2}-\sqrt{\rho_{N}}+2 K_{N}}{2+\sqrt{\rho_{N}} \cdot s_{N}^{2}-\sqrt{\rho_{N}}}} \cdot\left(\rho_{N}-1\right)}{\rho_{N}{ }^{2 \cdot \frac{\sqrt{\rho_{N}} \cdot s_{N}^{2}-\sqrt{\rho_{N}}+K_{N}+1}{2+\sqrt{\rho_{N}} \cdot s_{N}^{2}-\sqrt{\rho_{N}}}}-1}
$$

Similarly, for $N$ zones the utilization rate of the shuttle is given by

$\rho_{N}=\frac{t_{S_{N}}}{t_{A_{N}}}$

and the queueing system capacity for each zone of the $\mathrm{SBS} / \mathrm{RS}$ is

$K_{N}=n_{b u f_{N}}+1$

The third argument $s_{N}$, or the coefficient of variation of the service process has to be evaluated by a numerical simulation of the service times.

Respectively, the probability of emptiness contains the same arguments as the blocking probability and can be calculated for each zone of the SBS/RS through

$$
p_{0_{N}}=\frac{\rho_{N}-1}{2 \cdot \frac{\sqrt{\rho_{N}} \cdot s_{N}^{2}-\sqrt{\rho_{N}}+K_{N}+1}{2+\sqrt{\rho_{N}} \cdot s_{N}^{2}-\sqrt{\rho_{N}}}}-1
$$

\subsection{Zoning in vertical and horizontal direction}

The third zoning type treated in this paper is the twodimensional zoning, that is, zoning in the vertical and horizontal directions, as shown in Fig. 5.

For this zoning type, the throughput was calculated via a combination of equations for zoning in horizontal (see Section 4.2) and vertical (see Section 4.3) directions. Initially, a definition was necessary: The rack has different zones $\mathrm{N}$ with a relative amount of all storage locations $n_{N}$ 
Fig. 5 Zoning in vertical and horizontal direction

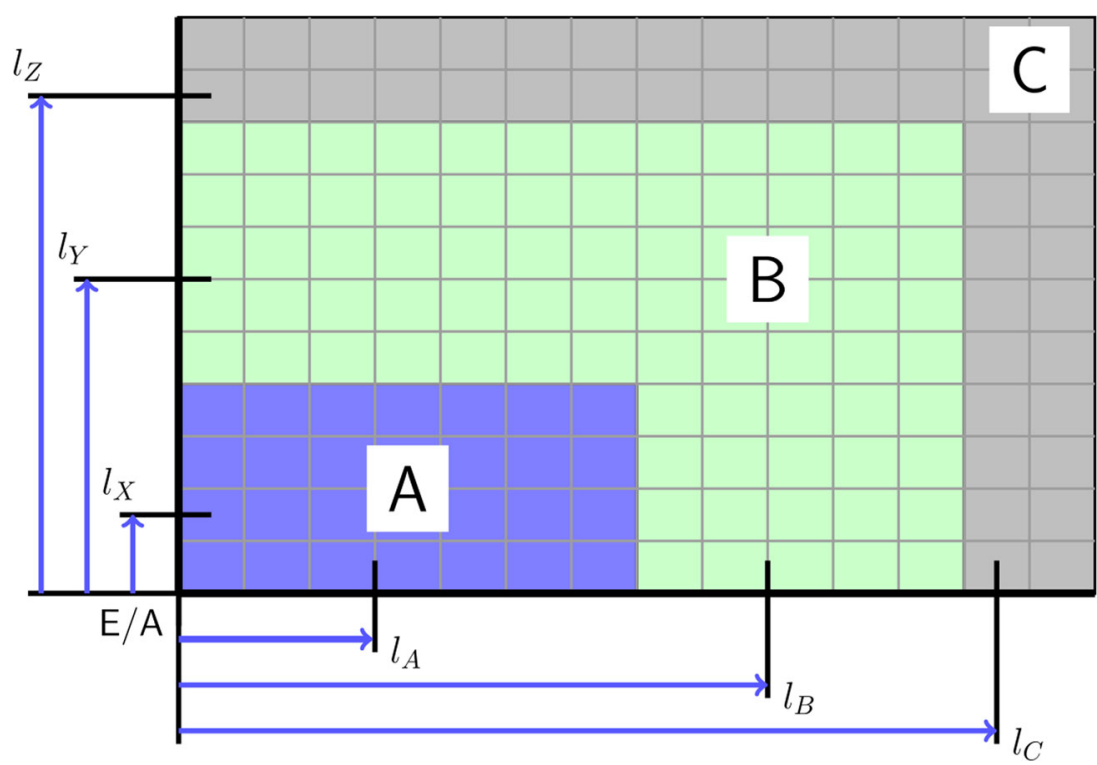

and a probability of order $w_{N}$. These are respectively the different classes of the storage policy. To transfer zones $N$ into different areas $X$ in the vertical direction, the probability $w_{X N}$ of being in area $\mathrm{X}$ was used. This factor $w_{X N}$ describes the probability that a tote from zone/class $N$ is situated in area $X$. The areas $X$ can have 1 to $n_{\text {tier }}$ tiers. For example, the rack shown in Fig. 5 has three zones $(A-C)$ and three areas $(\mathrm{X}-\mathrm{Z})$. The area $X$ at the bottom includes all of the rack with totes from class A and a part of class B and class $C$. In the figure, this area was marked with the mean height for the ride of the lifts $l_{X}$. The second area $Y$ above contained a part of classes $\mathrm{B}$ and $\mathrm{C}$, and was marked with the mean height to the ride of the lifts $l_{Y}$. The third area $Z$ only contained totes from class $\mathrm{C}$ and was marked with $l_{Z}$.

Accordingly, the number of tiers of the area $X$ can be calculated through

$n_{\text {tier }_{X}}=n_{\text {tier }} \cdot \sum_{N=1}^{\text {zones }} w_{N X} \cdot n_{N}$

which is the summation of the probabilities that a tote from zone/class $N$ is situated in area $X$ times the number of storage locations of class $N$. This sum is multiplied by the number of tiers to obtain the number of tiers in area $X$.

The second factor that has to be transferred is the probability of order from area $X$,

$w_{X}=\sum_{N=1}^{\text {zones }} w_{N X} \cdot w_{N}$

which is the sum of the probabilities that a tote from class $N$ is situated in area $X$ times the probability of order from zone/class $N$.
The third factor that has to be transferred is the number of slots for articles from zone $N$ in area $X$, that is, the distribution of the articles from the classes over the length of a tier.

$n_{\text {slot }_{N X}}=\frac{w_{N X} \cdot n_{N} \cdot n_{\text {slot }} \cdot n_{\text {tier }}}{n_{\text {tier }_{X}}}$

Equation 41 contains the probability that a tote from zone $N$ is situated in area $X$ multiplied by the number of storage slots from zone/class $N$, the number of slots on each side per aisle, and the number of tiers, and then all these terms are divided by the number of tiers in area $X$.

The last and important factor is the probability of order within a tier in the respective area $X$. This is governed by an expression converting the overall probability of order $w_{N}$ into the probability of order within on tier, for calculation of the service time of the respective tier,

$w_{X N}=\frac{w_{N} \cdot w_{N X}}{\sum_{N=1}^{z o n e s} w_{N} \cdot w_{N X}}$

Moreover, such conversion can be achieved by multiplication of the probability of order from zone/class $N$ with the probability that a tote from zone/class $N$ is situated in area $X$. The product is then divided by the sum over all zones of the same multiplication.

\subsubsection{Interarrival time}

Calculations for the interarrival times of the lift with two-dimensional zoning is quite similar to that for the interarrival times with zoning in vertical direction. The main difference, however, is that the calculation now uses different vertical areas $X$ with their number of tiers $n_{t i e r}$ and probability of selection $w_{X}$. 
Single-command cycle: The single-command cycle is governed by the equations

$$
\begin{aligned}
t_{\text {lift } t_{S C}=} & 2 \cdot t_{R_{L_{-} S C}}+t_{t_{L}} \\
t_{R_{L_{-} S C}=} & \sum_{X=1}^{\text {Areas }} w_{X} \cdot \frac{1}{n_{\text {tier } X}} \\
& \cdot \sum_{k=1}^{n_{\text {tier }}} \mathbf{t}\left((k-1) \cdot \Delta y+\sum_{M=0}^{N-1} n_{\text {tier }} \cdot \Delta y\right)
\end{aligned}
$$

Transformation of the cycle time of the lifts to the interarrival times into the different tiers of the respective areas:

$t_{A_{X}}=t_{l i f t} \cdot \frac{n_{\text {tier }}}{w_{X}}$

\subsubsection{Service time for a single depth rack}

The calculation of the service times of the shuttle for the two-dimensional zoning is quite similar to that for the service time with zoning in horizontal direction. The main difference is the different probabilities of selection within the respective tiers $w_{X N}$ and the number of slots assigned to the different zones $n_{\text {slot }_{N X}}$.

Single-command cycle: For the single-command cycle the equations are as follows.

$$
\begin{aligned}
& t_{S_{X}}=t_{\text {shuttle }_{S C_{X}}}=2 \cdot t_{R_{S} S C_{X}}+t_{t_{S}} \\
& t_{R_{S-S C_{X}}}=\sum_{N=1}^{\text {Zones }_{N=1}} w_{X N} \cdot \frac{1}{n_{\text {slot }_{N X}}} \\
& \cdot \sum_{k=1}^{n_{\text {slot }_{N}}} \mathbf{t}\left(k \Delta y+\sum_{M=0}^{N-1} n_{\text {slot }_{M X}} \cdot \Delta y\right)
\end{aligned}
$$

Dual-command cycle: For the dual-command cycle is defined through the equations.

$$
\begin{aligned}
& t_{S_{X}}=t_{\text {shuttle }_{D C_{X}}}=2 \cdot t_{R_{S-S C_{X}}}+t_{R_{S_{-} D C_{X}}}+2 \cdot t_{t_{S}} \\
& \sum_{J=1}^{t_{R_{S} D C_{X}}}= \\
& \sum_{l=1}^{\text {Oones }_{\text {Zones }}} \sum_{K=1}^{n_{\text {slot }} X} \frac{w_{X J}}{n_{\text {slot }_{J X}}} \frac{w_{X K}}{n_{\text {slot }_{K X} X}} \mathbf{t}\left(\mid\left(l+\sum_{O=0}^{J-1} n_{\text {slot }_{O}}-m-\sum_{P=0}^{K-1} n_{\text {slot }_{P}} \mid\right) \cdot \Delta x\right)
\end{aligned}
$$

\subsubsection{Open queueing model $\mathrm{M}|\mathrm{G}| 1 \mid \mathrm{K}$}

The calculation of the open queueing system with limited capacity of zoning in vertical direction is different from the calculation without zoning. With zoning in vertical direction the tiers are split up in different zones. Thus, the queueing system has to be calculated several times for each zone. Accordingly, the throughput of one single tier can be calculated through the subsequent equations, where $X$ is the number of the area.

$\vartheta_{\text {tier }_{X}}=\frac{1}{t_{A_{X}}} \cdot\left(1-p_{k_{X}}\right)$

$\vartheta_{\text {tier }_{X}}=\frac{1}{t_{S_{X}}} \cdot\left(1-p_{0_{X}}\right)$

Here, the throughput of an aisle is the summation over the different areas of the throughput of one tier in the respective area multiplied by the number of tiers in this area.

$\vartheta=\sum_{X=1}^{\text {Areas }} \vartheta_{\text {tier }_{X}} \cdot n_{\text {tier } X}$

Moreover, the blocking probability of a queueing system has to be calculated for each area $X$ through

$$
p_{k_{X}}=\frac{\rho_{X}^{\frac{\sqrt{\rho_{X}} \cdot s_{X}^{2}-\sqrt{\rho_{X}}+2 K_{X}}{2+\sqrt{\rho_{X}} \cdot s_{X}^{2}-\sqrt{\rho_{X}}}} \cdot\left(\rho_{X}-1\right)}{\rho_{X}{ }^{2 \cdot \frac{\sqrt{\rho_{X}} \cdot s_{X}^{2}-\sqrt{\rho_{X}}+K_{X}+1}{2+\sqrt{\rho_{X}} \cdot s_{X}^{2}-\sqrt{\rho_{X}}}}-1}
$$

Similarly, the utilization rate of the shuttle for the different areas $X$ can be calculated with

$\rho_{X}=\frac{t_{S_{X}}}{t_{A_{X}}}$

and capacity of the queueing system for each area of the SBS/RS can be calculated with the expression

$K_{X}=n_{b u f_{X}}+1$

The third argument, $s_{X}$, or the coefficient of variation of the service process has to be evaluated by a numerical simulation of the service times.

The probability of emptiness contains the same arguments as the blocking probability and can be calculated for each zone of the SBS/RS using

$$
p_{0_{X}}=\frac{\rho_{X}-1}{\rho_{X} \cdot \frac{\sqrt{\rho_{X} \cdot s_{X}^{2}}-\sqrt{\rho_{X}}+K_{X}+1}{2+\sqrt{\rho_{X}} \cdot s_{X}^{2}-\sqrt{\rho_{X}}}-1}
$$

\section{Numerical study}

Herein, the approach of the whole aisle with class-based storage was verified, wherefore the analytical model was verified via a comparison of the underlying assumptions of the time distributions with the results of the numerical simulation. Subsequently, an outline on how the model should be used to improve SBS/RS was drafted. The specific impact of the class-based storage policy was further demonstrated by an example. 
Table 3 Tested parameter configurations of the tier-captive single-aisle SBS/RS

\begin{tabular}{ll}
\hline Parameter & Value \\
\hline Number of tiers & $n_{\text {tier }}=40$ \\
Number of storage slots on each side of the aisle per tier & $n_{\text {slot }}=200$ \\
Number of buffers on each side of the aisle per tier & $n_{\text {buf }}=1$ \\
Distance between two storage slots & $\Delta x=0,5 m$ \\
Distance between two tiers & $\Delta y=0,4 m$ \\
Lift velocity & $v_{\text {lift }}=5 \frac{\mathrm{m}}{\mathrm{s}}$ \\
Lift acceleration/deceleration rate & $a_{\text {lift }}=7 \frac{\mathrm{m}}{\mathrm{s}^{2}}$ \\
Time to transfer a tote to and from the lift & $t_{t_{L}}=2.8 \mathrm{~s}$ \\
Shuttle velocity & $v_{\text {shuttle }}=2 \frac{\mathrm{m}}{\mathrm{s}}$ \\
Shuttle acceleration/deceleration rate & $a_{\text {shuttle }}=2 \frac{\mathrm{m}}{\mathrm{s}^{2}}$ \\
Time to transfer a tote to and from the shuttle & $t_{t_{S}}=8.4 \mathrm{~s}$ \\
Number of slot in zone N for 2 zones & $n_{N}=20 \% / 80 \%$ \\
Number of slot in zone N for 3 zones & $n_{N}=20 \% / 30 \% / 50 \%$ \\
Probability of order a tote in zone N for 2 zones & $w_{N}=60 \% / 40 \%$ \\
Probability of order a tote in zone N for 3 zones & $w_{N}=60 \% / 30 \% / 10 \%$ \\
\hline
\end{tabular}

\subsection{Numerical evaluation of the approximation quality}

During the design process of a SBS/RS, the performance of one aisle is important when designing the other parts of a storage system, such as a conveyor. Understanding the influence of class-based storage helps to determine an economically and ecologically ideal design of SBS/RS. Thus, herein, certain parameter configurations as shown in Table 3 were selected in order to present a variety of different settings. The system in discussion had 40 tiers and 200 storage slots on each side of the aisle. The parameters in Table 3 were specified by a European material handling provider of SBS/RS.

\subsubsection{Time distributions of the interarrival time}

The underlying assumption behind the open queueing model $\mathrm{M}|\mathrm{G}| 1 \mid \mathrm{K}$ states that the time distribution of the interarrival time by the lift is an exponential distribution. Eder's [2] approach assumed that all tiers have the same exponentially distributed interarrival time; thus, to fulfil this, the approach was divided in different parts. Figures 6 , 7,8 , and 9 show a class-based storage with three classes and zoning in the vertical direction. Figure 6 presents the arrivals into the different tiers, specifically, the three different zones with their probabilities of numbers of tiers into the respective zone $n_{N}=20 \% / 30 \% / 50 \%$ and with their probabilities of numbers from the respective zone
Fig. 6 Number of arrivals into the different tiers

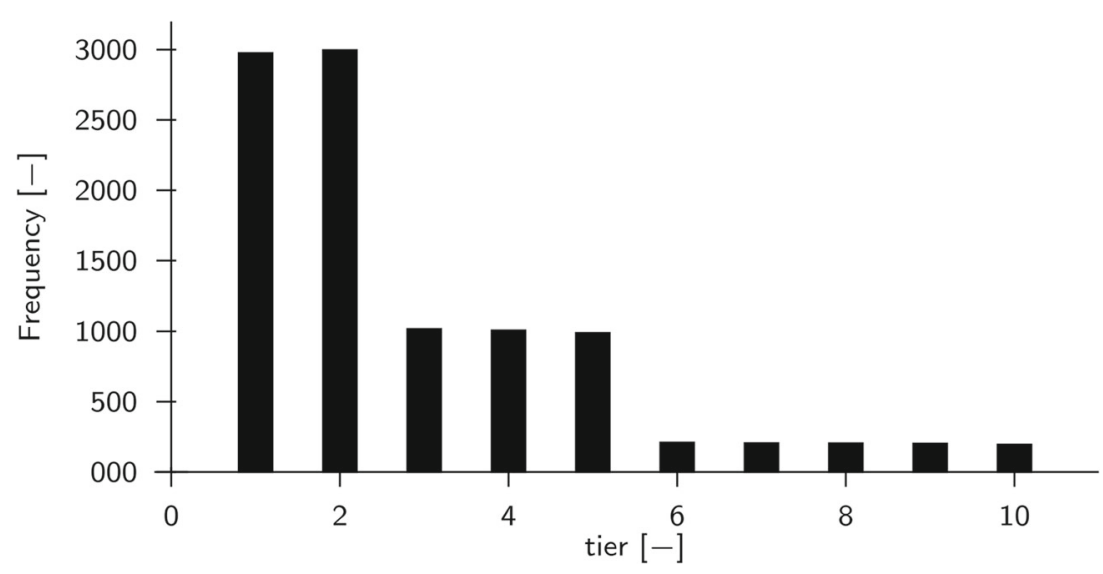


Fig. 7 Histogram of the

interarrival times to the tiers of zone A

Fig. 8 Histogram of the interarrival times to the tiers of zone B

Fig. 9 Histogram of the interarrival times to the tiers of zone C
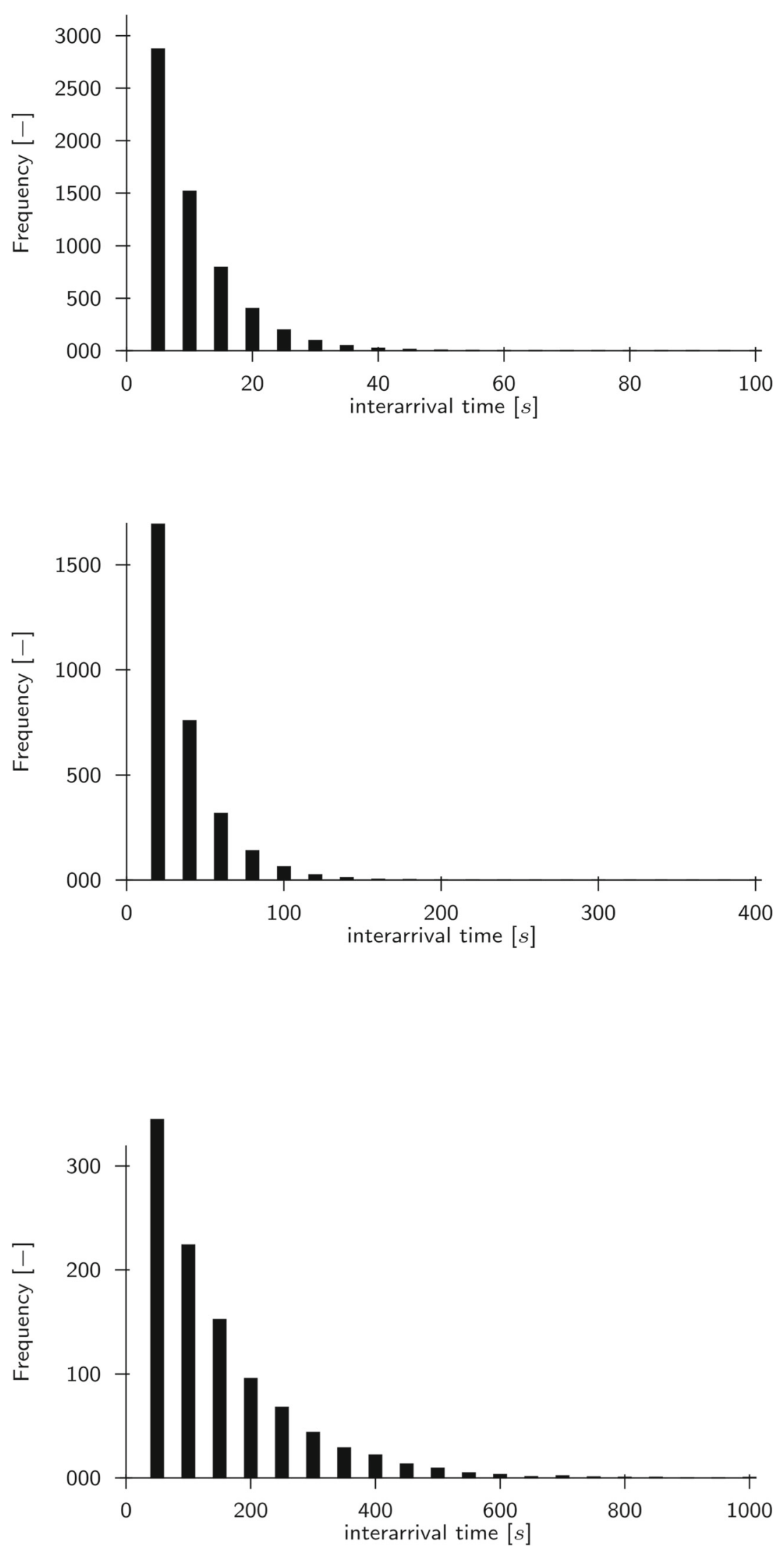
Fig. 10 Histogram of the maximum number of slots to ride during a double handling cycle

Fig. 11 Two zones in horizontal direction $\vartheta=482 \frac{\text { totes }}{h}$

Fig. 12 Two zones in vertical direction $\vartheta=495 \frac{\text { totes }}{h}$

Fig. 13 Two zones in horizontal and vertical direction with optimized length and width of the zones $\vartheta=536 \frac{\text { totes }}{h}$

Fig. 14 Three zones in horizontal and vertical direction with optimized length and width of the zones $\vartheta=1003 \frac{\text { totes }}{h}$
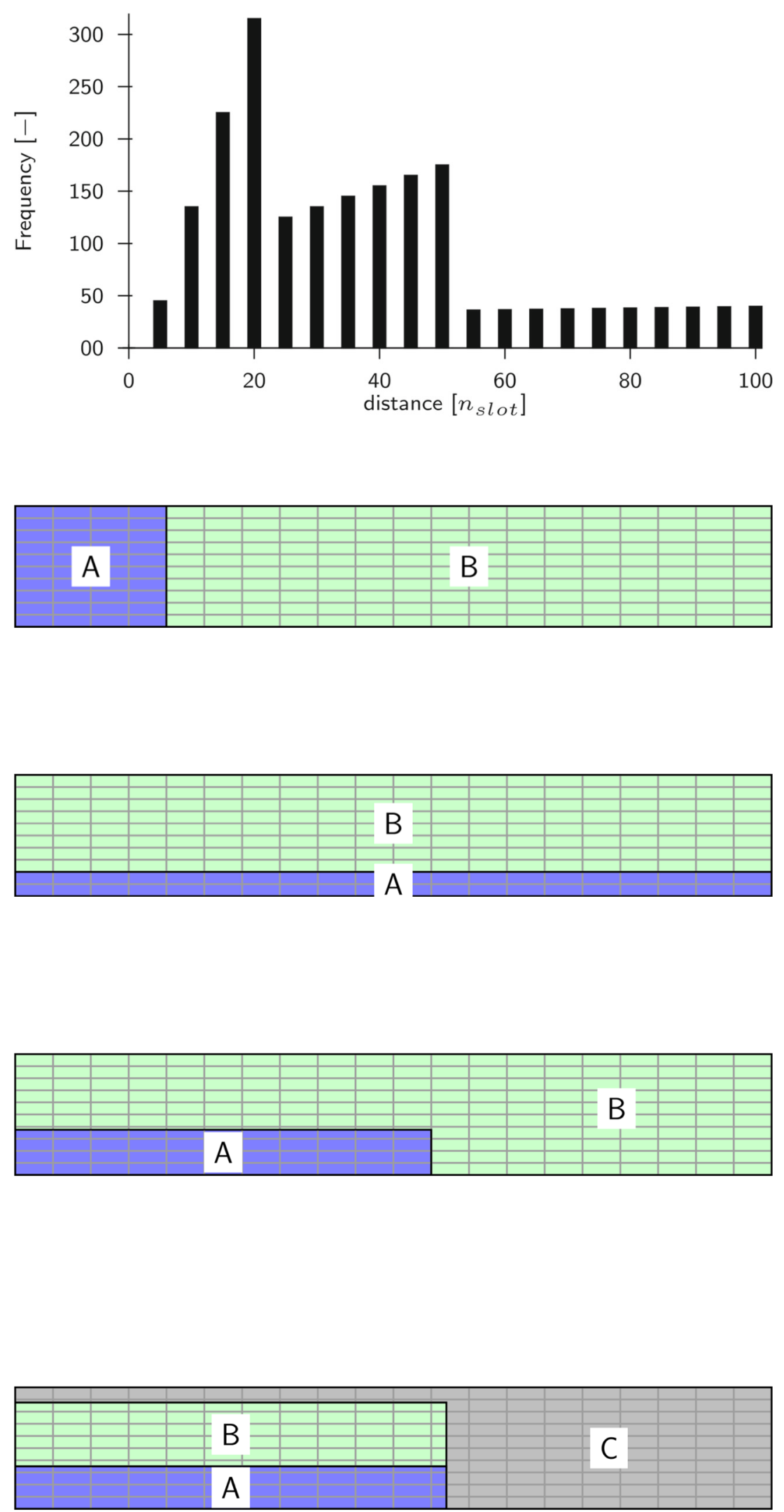
$w_{N}=60 \% / 30 \% / 10 \%$. The next three diagrams present the histograms of the interarrival times to the tiers in zone A (Fig. 7), zone B (Fig. 8), and zone C (Fig. 9). All these distributions correspond to an exponential distribution with a mean interarrival time for the zones as follows: zone $\mathrm{A}$ $t_{A_{A}}=8.2 \mathrm{~s}$, zone B $t_{A_{B}}=24.5 \mathrm{~s}$ and zone $\mathrm{C} t_{A_{C}}=124.7 \mathrm{~s}$.

\subsubsection{Time distribution of the service time}

For the service time of shuttles in the SBS/RS, Eder [2] assumed that the maximum distance to ride within one double handling cycle is represented by a rightskewed triangular distribution. This is appropriate upon simplification where the ride times are calculated using Eq. 4. The coefficient of variation of such a distribution without the constant transfer times is approximately $s=$ 0.57 . For the class-based storage, this assumption should be changed. For such a case, the distribution of the maximum ride distance within one double handling cycle is a combination of three right-skewed triangular distributions as shown in Fig. 10 with the peaks of the triangles becoming lower with increase of zone position. Usually, this occurs at a higher coefficient of variation, such as for about $s=0.78$ without transfer times. The underlying assumption for this distribution of ABC-zoning was $w_{N}=60 \% / 30 \% / 10 \%$ and $n_{N}=20 \% / 30 \% / 50 \%$. Also, the mean distance to ride in a double handling cycle with an aisle length of $n_{\text {slot }}=200$, changed from $n_{\text {mean }}=133$ without zoning to $n_{\text {mean }}=36$ at the three class-zoning. Consequently, the real distribution had to be simulated numerically in order to obtain the right coefficient of variation.

\subsection{Optimization example}

The optimization example was based on the parameters in Table 3. The system discussed was a SBS/RS with 16000 spaces, 400 slots per tier and 40 tiers. A system with these parameters and without zoning would reach a throughput of $\vartheta=473 \frac{\text { totes }}{h}$. The first option for zoning with two classes was zoning in the horizontal direction, as depicted in Fig. 11. This kind of zoning increased the reachable throughput to $\vartheta=482 \frac{\text { totes }}{h}$. The second option was zoning in the vertical direction, as indicated in Fig. 12, which reached a throughput of $\vartheta=495 \frac{\text { totes }}{h}$. The next step was zoning in the vertical and horizontal directions with a rectangle block of zone A, as demonstrated in Fig. 13, which reached a throughput of approximately $\vartheta=536 \frac{\text { totes }}{h}$

. With this optimal configuration, zone A had a length of $n_{\text {slot }_{A}}=110$ and height of $n_{\text {tier }_{A}}=15$. Figure 14, on the other hand, shows the possible scenarios with the same physical configuration, but with zoning in three zones. Such configuration yielded a throughput of $\vartheta=1003 \frac{\text { totes }}{h}$. The length of zone A was $n_{\text {slot }_{A}}=114$ and its height was $n_{\text {tier }}=14$. Likewise, the length of zone B was $n_{\text {slot }_{B}}=$ 114 and its maximum height was $n_{\text {tier }_{B}}=35$. Apparently, for this example the transfer from two zones to three zones showed nearly double throughput.

This optimization showed the possible scenarios with an existing system with applying a class-based storage policy. For the example, the SBS/RS was provided with all the physical parameters that were constant. Thus, the only way to improve the performance is by modifying the storage policy to a class-based one. Depending on how the order list is composed, different storage policies can be applied. The reachable throughput reaches from $\vartheta=473 \frac{\text { totes }}{h}$ without zoning to $\vartheta=1003 \frac{\text { totes }}{h}$ for zoning in three zones, with zoning in the vertical and horizontal directions.

\section{Conclusion}

The high system performance of tier-captive SBS/RS has been increasingly sought by industries. To ameliorate the performance even further, a different storage policy such as a class-based storage, can be employed. Nevertheless, there are few available decision tools for evaluating such performance, a discrete event simulation being the most frequently used. Thus, this study presented a method for calculating the performance of tier-captive single-aisle SBS/RS that equally delivers accurate results. The system was modelled as a continuous-time open queueing system with limited capacity. Subsequently, the interarrival and service times were evaluated using the cycle time model in combination with a probability based approach of lifts and shuttles with discrete spatial values. The presented approach has the distinct feature that it can be used to discuss a high number of different configurations of class-based storage in a very fast and accurate manner. The accuracy of the analytical model was achieved by extraction of the different time distributions of the interarrival and service times and by comparing them with the assumptions for the continuoustime open queueing model with limited capacity. The main novelty of this approach is the analysis of the interactions between shuttles and lifts with the help of a limited-capacity open queueing model $(\mathrm{M}|\mathrm{M}| 1 \mid \mathrm{K})$ in combination with a class-based storage policy. This provides the possibility of determining the throughput of various configurations of SBS/RS with class-based storage policies that are governed by a variety of physical parameters, such as the following: the length or the height of the rack, as well as various classbased storage policy variants, such as the number of classes in the system. In general, the presented approach reaches a high approximation quality. Finally, an outline was provided on how the presented approach can be used to optimize an existing tier captive SBS/RS. An example demonstrated the influence of different storage policies and the throughput 
that can be achieved by optimizing the storage policy. Moreover, this approach can be used to optimize zoning of different storage policies, which is an advantage for any provider of SBS/RS. The presented assumptions were similar to the SBS/RS of a European material handling provider. Further work will be dedicated to the SBS/RS with class-based storage to evaluate a calculation method for determining the places of the various zones along the rack. The zone spread discussed in this article is set to rectangular zones. In further work, it must be examined if this is the best spread or if other types of areas provide a higher throughput. Another aspect that needs to be addressed is the extension of the developed approach to multiple-deep storage in order to achieve higher storage densities of the SBS/RS.

Funding information Open access funding provided by TU Wien (TUW). This work was supported by the TU Wien University Library, through its Open Access Funding Programme.

\section{Compliance with ethical standards}

Conflict of interests The author declares that he has no conflict of interest.

Open Access This article is licensed under a Creative Commons Attribution 4.0 International License, which permits use, sharing, adaptation, distribution and reproduction in any medium or format, as long as you give appropriate credit to the original author(s) and the source, provide a link to the Creative Commons licence, and indicate if changes were made. The images or other third party material in this article are included in the article's Creative Commons licence, unless indicated otherwise in a credit line to the material. If material is not included in the article's Creative Commons licence and your intended use is not permitted by statutory regulation or exceeds the permitted use, you will need to obtain permission directly from the copyright holder. To view a copy of this licence, visit http:// creativecommonshorg/licenses/by/4.0/.

\section{References}

1. Carlo HJ, Vis IF (2012) Sequencing dynamic storage systems with multiple lifts and shuttles. Int J Prod Econ 140(2):844-853

2. Eder M (2019) An analytical approach for a performance calculation of shuttle-based storage and retrieval systems. Production \& Manufacturing Research 7(1):255-270. https://doi.org/10.1080/21693277.2019.1619102
3. Eder M, Kartnig G (2016) Durchsatzoptimierung von shuttleSystemen mithilfe eines analytischen Berechnungsmodells Logistics journal: Proceedings 2016(10)

4. Eder M, Kartnig G (2016) Throughput analysis of s/r shuttle systems and ideal geometry for high performance. FME Trans 44(2):174-179

5. Eder M, Kartnig G (2018) Calculation method to determine the throughput and the energy consumption of $\mathrm{s} / \mathrm{r}$ shuttle systems. FME Trans 46(3):424-428

6. Ekren B, Sari Z, Lerher T (2015) Warehouse design under classbased storage policy of shuttle-based storage and retrieval system. IFAC-PapersOnLine 48(3):1152-1154

7. Epp M, Wiedemann S, Furmans K (2017) A discrete-time queueing network approach to performance evaluation of autonomous vehicle storage and retrieval systems. Int J Prod Res 55(4):960978

8. Heragu SS, Cai X, Krishnamurthy A, Malmborg CJ (2011) Analytical models for analysis of automated warehouse material handling systems. Int J Prod Res 49(22):6833-6861

9. Kriehn T, Schloz F, Wehking KH, Fittinghoff M (2018) Impact of class-based storage, sequencing of retrieval requests and warehouse reorganisation on throughput of shuttle-based storage and retrieval systems. FME Trans 46(3):320-329

10. Kuo PH, Krishnamurthy A, Malmborg CJ (2008) Performance modelling of autonomous vehicle storage and retrieval systems using class-based storage policies. Int J Comput Appl Technol 31(3-4):238-248

11. Lerher $\mathrm{T}$ (2013) Modern automation in warehousing by using the shuttle based technology. Automation Systems of the 21st century: New Technologies, Applications and Impacts on the Environment \& Industrial Processes pp 51-86

12. Lerher T, Edl M, Rosi B (2014) Energy efficiency model for the mini-load automated storage and retrieval systems. The Int J Adv Manuf Technol 70(1-4):97-115

13. Marchet G, Melacini M, Perotti S, Tappia E (2012) Analytical model to estimate performances of autonomous vehicle storage and retrieval systems for product totes. Int $\mathrm{J}$ Prod Res 50(24):7134-7148

14. Marchet G, Melacini M, Perotti S, Tappia E (2013) Development of a framework for the design of autonomous vehicle storage and retrieval systems. Int J Prod Res 51(14):4365-4387

15. Schloz F, Kriehn T, Wehking KH, Fittinghoff M (2017) Entwicklung situationsabhängiger lagerstrategien für hochregallager mit autonomen fahrzeugen Logistics Journal: Proceedings 2017(10)

16. Smith JM (2004) Optimal design and performance modelling of m/G/1/K queueing systems. Math Comput Model 39(9-10):1049_ 1081

Publisher's note Springer Nature remains neutral with regard to jurisdictional claims in published maps and institutional affiliations. 